\title{
Stereosequence Distributions of Poly(t-butyl methacrylate)s Prepared with Butyllithium
}

\author{
Toshimitsu Suzuki, Osamu Yamada, Yoshihisa Watanabe, \\ and Yoshinobu TAKEGAMI \\ Department of Hydrocarbon Chemistry, Faculty of Engineering, \\ Kyoto University, Kyoto 606, Japan.
}

(Received September 30, 1981)

\begin{abstract}
C}$ NMR spectra of poly( $t$-butyl methacrylate)s (PBMA) prepared with butyllithium $(n-\mathrm{BuLi})$ were studied. Methylene carbon resonances appeared as five overlapped peaks, which were interpreted as a combination of hexad stereosequence distributions. The stereosequence distribution of PBMA prepared with $n$-BuLi at $-78^{\circ} \mathrm{C}$ obeyed the first order Markov chain model even polymerized in tetrahydrofuran (THF). Syndiotactic PBMA was obtained with azobisisobutyronitrile (AIBN) as the initiator, and the stereosequence distribution of this polymer agreed well with Bernoullian statistics.
\end{abstract}

KEY WORDS ${ }^{13} \mathrm{C}$ NMR / Poly( $t$-butyl methacrylate) / Microtacticity / Anionic polymerization / Butyllithium

A large number of studies on the stereosequence distributions of vinyl polymers have been carried out using ${ }^{1} \mathrm{H}$ and ${ }^{13} \mathrm{C}$ NMR spectroscopy, and the results are summarized in the literature. ${ }^{1,2}$

The stereosequence distribution of poly(methyl methacrylate) (PMMA) was established up to pentad sequences using high field ${ }^{1} \mathrm{H}$ and ${ }^{13} \mathrm{C}$ NMR spectroscopy. ${ }^{1,3}$ In the ${ }^{13} \mathrm{C}$ NMR spectrum of PMMA,$\alpha-\mathrm{CH}_{3}$ and quaternary carbon resonances give triad stereosequences and the carbonyl carbon resonance provides pentad stereosequences. ${ }^{3}$ However, the methylene carbon resonance was observed as a broad envelope of overlapped several resonances. Hatada et al. ${ }^{4}$ reported the splitting of the methylene carbon resonances up to the hexad stereosequence using a $90 \mathrm{MHz}{ }^{13} \mathrm{C} \quad \mathrm{NMR}$ spectrometer.

The effects of the ester group on the stereoregularity of poly(methacrylate)s prepared with anionic initiators have been studied by Tsuruta et al. ${ }^{5}$ and Yuki et al. ${ }^{6 a}$

At the time we carried out the synthesis of a block copolymer of poly(t-butyl methacrylate) and poly(ethylene oxide) initiated with macroanions, ${ }^{7}$ we found that the methylene carbon resonance in the ${ }^{13} \mathrm{C}$ NMR spectrum of the copolymer could be resolved into five peaks at $25.0 \mathrm{MHz}$. In this paper, we describe stereosequence distributions of poly $(t-$ butyl methacrylate) (PBMA) prepared under various conditions.

\section{RESULTS AND DISCUSSION}

Table I summarizes the polymer samples used in this study. ${ }^{13} \mathrm{C}$ NMR spectra were recorded on a JEOL FX-100 FT NMR spectrometer at $25.0 \mathrm{MHz}$. Approximately $15-20 \mathrm{wt} \%$ polymer solutions in $\mathrm{CDCl}_{3}$ were used for the measurement at $46^{\circ} \mathrm{C}$. Proton noise decoupling was employed throughout the experiment, and the peak areas were measured by cutting the spectrum traces and weighing.

Figure 1 shows typical spectra of PBMA. The ${ }^{13} \mathrm{C}$ NMR spectrum of PBMA prepared with $n$-BuLi in toluene shows a very simple pattern characteristic to a highly isotactic polymer. Unlike PMMA, the strong peaks due to the three carbons of the $t$-butyl group and due to an alkoxy quaternary carbon at 80 ppm could be observed without and with fine structure, respectively. These spectra differ from those of PMMA in the carbonyl carbon and methylene carbon resonances. In the carbonyl carbon resonance of PMMA, except for the heterotactic 


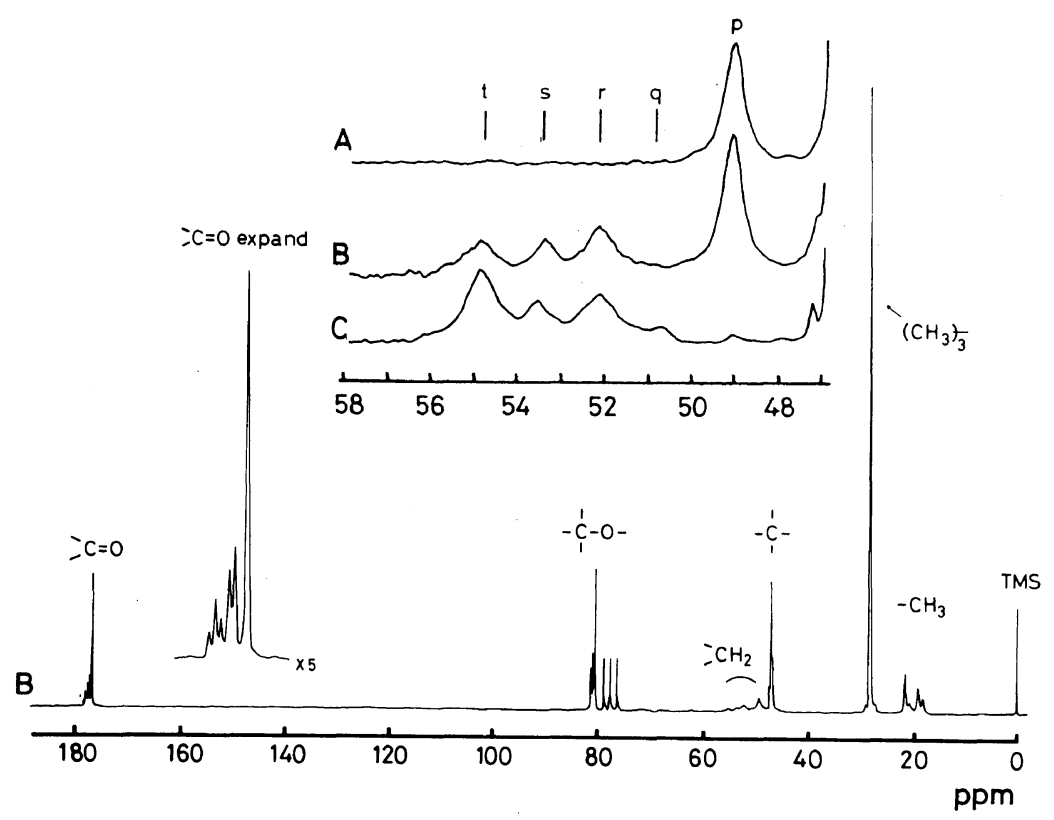

Figure 1. ${ }^{13} \mathrm{C}$ NMR spectrum of PBMA and expanded methylene carbon resonances: (A) sample No. 1; (B) sample No. 3; (C) sample No. 5. Assignment of peaks $\mathrm{p}-\mathrm{t}$, see Table II.

Table I. Polymerization of $t$-butyl methacrylate $^{\mathrm{a}}$

\begin{tabular}{|c|c|c|c|c|c|}
\hline \multirow{2}{*}{ Sample } & \multirow{2}{*}{ Initiator } & \multirow{2}{*}{ Solvent } & Temp & Time & Yield \\
\hline & & & ${ }^{\circ} \mathrm{C}$ & $\mathrm{h}$ & $\%$ \\
\hline 1 & $n$-BuLi & Toluene & -78 & 1 & 23 \\
\hline 2 & $n$-BuLi & Toluene & 0 & 2 & 92 \\
\hline 3 & $n-\mathrm{BuLi}$ & THF & -78 & 6 & 55 \\
\hline 4 & $n$-BuLi & THF & 0 & 3 & 100 \\
\hline $5^{b}$ & AIBN & & 60 & 1 & 23 \\
\hline
\end{tabular}

a Monomer, $8 \mathrm{mmol}$; $n$-BuLi, $0.3 \mathrm{mmol}$; solvent, $10 \mathrm{ml}$.

b Monomer, $10 \mathrm{mmol}$; AIBN, $0.1 \mathrm{mmol}$; in the bulk.

triad, well-resolved pentad stereosequence distributions could be obtained. However, in PBMA, an $\mathrm{mm}$ triad could not be separated into pentad stereosequences. PBMA prepared with $n$-BuLi in THF at $-78^{\circ} \mathrm{C}$ (sample No. 3) was unexpectedly rich in a meso dyad $^{6 \mathrm{~b}}$ and the stereosequence distribution of this polymer can be interpreted in terms of the first order Markov chain model shown in Table II. This is quite different from the case of PMMA. Similar results have been reported by Yuki et al. in regard to the polymerization of trityl methacrylate. ${ }^{6 a}$ When the polymerization temperature was $0^{\circ} \mathrm{C}$, the amount of racemic dyad increased and the stereosequence distribution obeyed the first order Markov chain model with $P_{m / m}=0.319$ and $P_{m / r}=$ 0.359 .

In general, when methyl methacrylate (MMA) is allowed to polymerize anionically at a low temperature, more racemic dyads appear in both toluene and THF than in the polymerization at room temperature. This closely related to the temperature dependence of the fraction of solvent (or agent) separated ion pairs. As mentioned above, the exceptional behavior of BMA in the anionic polymerization in THF cannot be accounted for easily. On the other hand, as indicated by Yuki et al. ${ }^{6 \mathrm{a}, \mathrm{c}}$ the steric control in the anionic polymerization of various alkyl methacrylate may not simply be attributed to the bulkiness of the ester group. However, in the polymerization of BMA, the strong interaction of the counter lithium cation and the growing chain end may favour isotactic placement of the next monomer. At $0^{\circ} \mathrm{C}$ in THF, the interaction between the counter cation and the living chain end decreases to give atactic PBMA.

Methylene carbon resonances of PBMA appeared as five overlapped peaks. These resonances 
Stereosequence Distributions of Poly( $t$-butyl methacrylate)s

Table II. Observed and calculated stereosequence distributions of poly( $t$-butyl methacrylate)

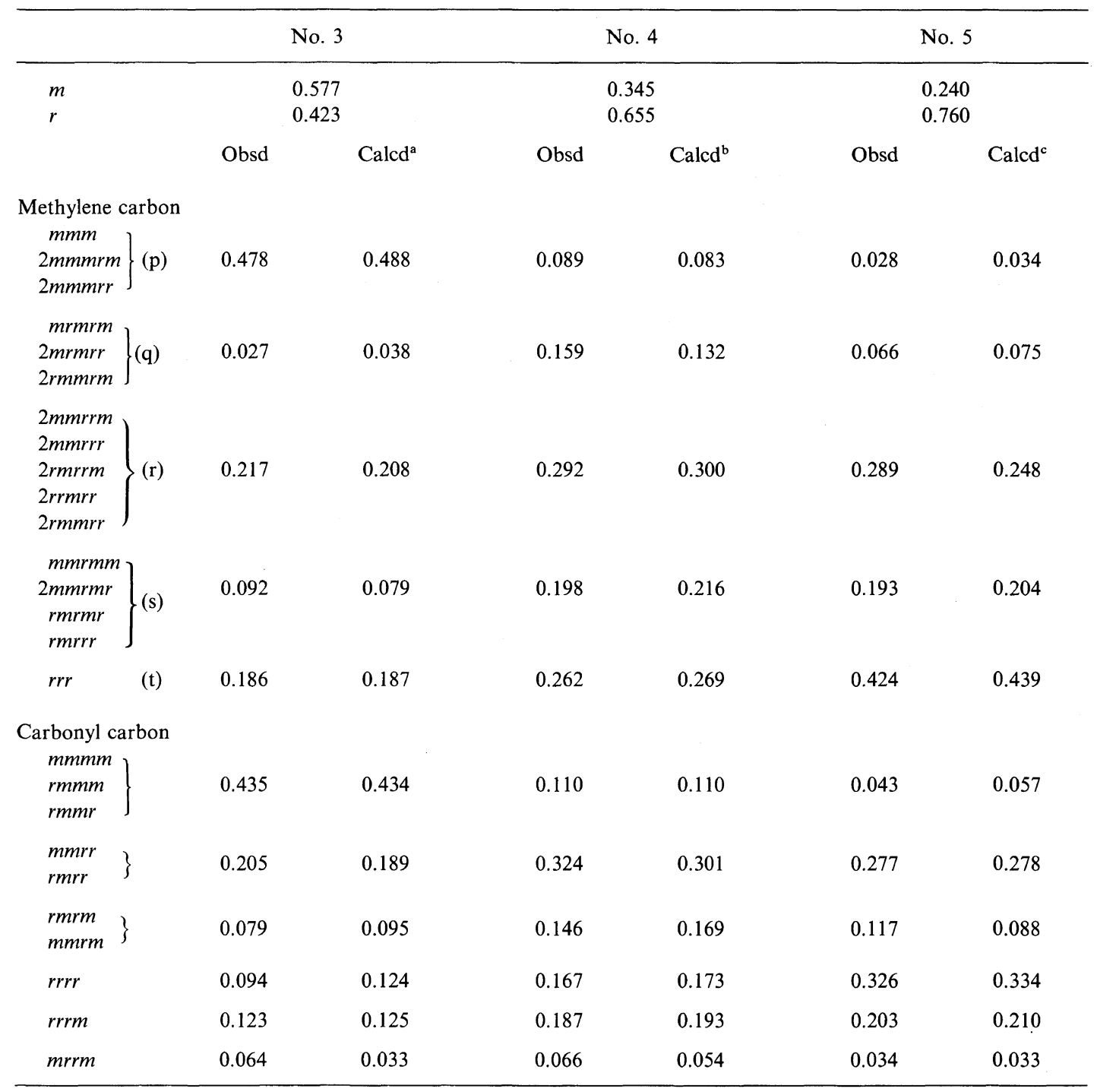

a 1st order Markov chain model, $P_{m / m}=0.754, P_{r / r}=0.644$.

b 1st order Markov chain model, $P_{m / m}=0.319, P_{r / r}=0.641$.

c Bernoullian statistics, $P_{m}=0.240$.

(p)-(t) correspond to the methylene carbon resonances shown in Figure 1.

cannot simply be interpreted as arising from tetrad stereosequence distributions. Polymer sample No. 1 , which is purely isotactic, exhibits a single peak at $49.0 \mathrm{ppm}$. Therefore, the highest methylene carbon resonance can be ascribed to a meso centered placement. In polymers No. 3, No. 4, and No. 5, the relative intensity of the observed peak at $49.0 \mathrm{ppm}$ falls considerably short of the $\mathrm{mmm}$ tetrads calculated from the first order Markov chain model or Bernoullian statistics. Furthermore, no combination of calculated tetrad sequences fits the observed results. However, the lowest field resonance of the methylene carbon at $54.8 \mathrm{ppm}$ may be assigned to the $r r$ tetrad, since good agreement between the 
calculated and observed intensity was obtained.

Hexad stereosequence distributions were calculated for samples No. 3 and No. 4 from the first order Markov chain model and for No. 5 from Bernoullian statistics. By comparing the observed values with the calculated hexad stereosequence distributions, four peaks of the methylene carbon resonance were tentatively assigned as shown in Table II. The principle used in this assignment is that hexad sequences rich in $r$ units shift down-field compared to those rich in $m$ units. Due to the appreciable overlapping of the peaks, measurement accuracy is not high. In the case of PMMA, the methylene carbon resonances appeared in the range from 52.0 to $55.5 \mathrm{ppm}^{4}$ The splitting of the spectrum for PBMA, however, was observed over more than $6 \mathrm{ppm}$, ranging from 49.0 to $55.0 \mathrm{ppm}$. This may be due to the differences between the conformation of PBMA and that of PMMA in solution.

PBMA prepared by a radical initiator was rich in the syndiotactic dyad and its stereosequence distribution was interpreted by Bernoullian statistics.

\section{REFERENCES}

1. F. A. Bovey, "High Resolution NMR of Macromolecules," Academic Press, New York, 1972.

2. J. C. Randall, "Polymer Sequence Determination," Academic Press, New York, 1977.

3. Y. Inoue, A. Nishioka, and R. Chujo, Polym. J., 4, 535 (1971).

4. K. Hatada, Kobunshi (High Polymers, Japan), 30, 696 (1981).

5. T. Tsuruta, M. Makimoto, and H. Kanai, J. Macromol. Chem., 1, 31 (1966).

6. a) H. Yuki, K. Hatada, K. Ohta, and Y. Okamoto, J. Macromol. Sci.-Chem., A9, 983 (1975).

b) K. Hatada, T. Kitayama, Y. Okamoto, K. Ohta, Y. Umemura, and H. Yuki, Makromol. Chem., 179, 485 (1978).

c) H. Yuki, Y. Okamoto, Y. Shimada, K. Ohta, and K. Hatada, J. Polym. Sci., Polym. Chem. Ed., 17, 1215 (1979).

7. T. Suzuki, O. Yamada, Y. Murakami, Y. Takegami, and Y. Watanabe, Macromolecules, in press. 